TITLE:

\title{
Spin-triplet proton-neutron pair in spin-dipole excitations
}

AUTHOR(S):

Yoshida, Kenichi; Tanimura, Yusuke

CITATION:

Yoshida, Kenichi ...[et al]. Spin-triplet proton-neutron pair in spin-dipole excitations. Physical Review C 2021, 104(1): 014319.

ISSUE DATE:

2021-07

URL:

http://hdl.handle.net/2433/268041

RIGHT:

(C) 2021 American Physical Society 


\title{
Spin-triplet proton-neutron pair in spin-dipole excitations
}

\author{
Kenichi Yoshida $\mathbb{D}^{*}$ \\ Department of Physics, Kyoto University, Kyoto 606-8502, Japan \\ Yusuke Tanimura ${ }^{\circ}$ \\ Department of Physics, Tohoku University, Sendai 980-8578, Japan \\ and Graduate Program on Physics for the Universe, Tohoku University, Sendai 980-8578, Japan
}

(Received 14 April 2021; revised 18 June 2021; accepted 16 July 2021; published 27 July 2021)

\begin{abstract}
Background: Spin-triplet $(S=1)$ proton-neutron $(p n)$ pairing in nuclei has been under debate. It is well known that the dynamical pairing affects the nuclear matrix element of the Gamow-Teller (GT) transition and the double $\beta$ decay.

Purpose: We investigate the effect of the pn-pair interaction in the $T=0, S=1$ channel on the low-lying spin-dipole (SD) transition. We then aim at clarifying the distinction of the role in between the SD and GT transitions.

Method: We perform a three-body model calculation for the transition ${ }^{80} \mathrm{Ni} \rightarrow{ }^{80} \mathrm{Cu}$, where ${ }^{78} \mathrm{Ni}$ is taken as a core. The strength of the pair interaction is varied to see the effect on the SD transition-strength distribution. To fortify the finding obtained by the three-body model, we employ the nuclear energy-density functional method for the SD transitions in several nuclei, where one can expect a strong effect.

Results: The effect of the $S=1 p n$-pair interaction depends on the spatial overlap of the $p n$ pair and the angular momentum of the valence nucleons; the higher the angular momentum of the orbitals, the more significant the effect.

Conclusions: The dynamical $S=1$ pairing is effective even for SD states, although the spatial overlap of the $p n$ pair can be smaller than GT states. The SD transition involving high- $\ell$ orbitals with the same principal quantum number is strongly affected by the dynamical $S=1$ pairing.
\end{abstract}

DOI: 10.1103/PhysRevC.104.014319

\section{INTRODUCTION}

Pairing is a ubiquitous many-body correlation emerging in various systems, including a nuclear system [1]. The superfluidity or superconductivity is mostly understood by the spin-singlet pairing [2]. New facets of the nuclear pairing show up as a spatially correlated two-neutron in dilute and weak-binding systems [3-10]. The spatial localization of two neutrons has been confirmed experimentally [11,12]. Meanwhile, there has been an enduring discussion on another type of exotic unconventional pairing in nuclei: the ${ }^{3} S_{1}$ correlation of isoscalar (IS) proton-neutron $(p n)$ pairs in $N \approx Z$ nuclei [13]. The emergence of the $S=1 p n$-pair condensation is still controversial.

The fluctuation of the $S=1$ pair field and its effect on the observables has been discussed recently by investigating, e.g., the pn-pair transfer-type modes of excitation [14-16] and the spin susceptibility [17]. Furthermore, it has been known that the dynamical $S=1$ pairing affects the low-lying GamowTeller (GT) strengths in neutron-deficient nuclei [18-20] and lowers the GT states in energy and thus shortens the $\beta$-decay

\footnotetext{
"kyoshida@ ruby.scphys.kyoto-u.ac.jp
}

half-lives of neutron-rich nuclei [21-24] including deformed nuclei [25-27].

The effects of the $S=1$ pair interaction on the GT transition strengths have been thoroughly studied in $N=Z$ odd-odd nuclei with a three-body model of two nucleons around a spherical core [28-30]. A remarkable feature found in $N=Z$ odd-odd nuclei with an $L S$-closed core $\left({ }^{4} \mathrm{He},{ }^{16} \mathrm{O},{ }^{40} \mathrm{Ca}\right)$ is the appearance of the low-energy state with a strong GT strength. A similar finding is obtained by employing a nuclear energydensity functional (EDF) method [31]. The low-energy GT states have been indeed identified experimentally in the transitions of ${ }^{18} \mathrm{O} \rightarrow{ }^{18} \mathrm{~F}$ [32] and ${ }^{42} \mathrm{Ca} \rightarrow{ }^{42} \mathrm{Sc}[33,34]$.

The spin-dipole (SD) excitation is induced by the spin operator, similarly to the GT excitation. To the best of the authors' knowledge, however, the effects of the $S=1$ pair interaction on the SD transition strengths have not been well studied. The principal quantum numbers of the single-particle orbital of a proton and a neutron differ by one unit contrary to $\Delta N=0$ in the GT transition. Therefore, due to the imbalanced Fermi levels of neutrons and protons, the high-energy first-forbidden $\beta$ decay occurs in neutron-rich nuclei: $-1 \hbar \omega_{0}$ excitation [35]. The dynamical $S=1$ pair interaction has been considered for describing the $\mathrm{SD}$ excitation as well as the GT excitation in the systematic calculations [36-39]. Thus, it is necessary to investigate in details the role of the $S=1$ 
pair interaction in the forbidden transitions in order to gain a deeper understanding of the $\beta$-decay properties and to be able to make more accurate predictions, as well as to obtain a new aspect of the $S=1$ pairing.

Therefore, in this article, we are going to investigate the role of the $S=1 p n$-pair interaction in the SD transitions: how much and in which nuclei the dynamical $S=1$ pairing affects the SD excitation. To this end, we employ a three-body model to obtain an essential feature of the $S=1$ pairing in the SD excitation. To make what we find in the three-body model analysis solid and secure, we further perform the nuclear density-functional theory (DFT) calculation; a nuclear EDF method is utilized, which is a theoretical model being capable of handling nuclides with arbitrary mass numbers in a single framework [40,41].

This paper is organized in the following way. Three-body model analysis is performed in Sec. II, where the low-lying SD $1^{-}$state in ${ }^{80} \mathrm{Cu}$ is studied. Section III is devoted to the discussion by employing the nuclear EDF method to fortify the findings obtained by the three-body model calculation. Then, a summary is given in Sec. IV.

\section{THREE-BODY MODEL ANALYSIS}

In this section, we employ the three-body model to make a qualitative analysis on the effect of the $S=1 \mathrm{pn}$-pair interaction on the low-lying SD $1^{-}$states. Based on the analytic form of the matrix element of the $S=1$ interaction, we discuss the relation between the quantum numbers of single-particle states involved in the transition and the gain of energy due to the $S=1$ interaction. Here, we take the ${ }^{80} \mathrm{Ni} \rightarrow{ }^{80} \mathrm{Cu}$ transition as a target of the investigation. The SD states with $J=0$ and 1 appear lower than the GT states in energy in ${ }^{80} \mathrm{Cu}$, and these negative-parity states play an important role in determining the $\beta$-decay half-life, as pointed out in Refs. $[39,42]$.

\section{A. Three-body model}

The details of the model are described in Refs. $[4,9,28]$. We thus briefly recapitulate the basic equations relevant to the present study. The Hamiltonian of the present model is given as

$$
H=\sum_{i=1,2}\left[\frac{\boldsymbol{p}_{i}^{2}}{2 m}+V_{N c}\left(r_{i}\right)\right]+\frac{1}{2} \sum_{i \neq j} V_{N N}\left(\boldsymbol{r}_{i}, \boldsymbol{r}_{j}\right),
$$

where $m$ is the mass of a nucleon, $r=|\boldsymbol{r}|$, and the recoil motion of the core is neglected. The potential between the nucleon $(N)$ and the core nucleus $(c), V_{N c}$, is given by

$$
V_{N c}(r)=\left[V_{0}+V_{l s} r_{0}^{2}(\ell \cdot s) \frac{1}{r} \frac{d}{d r}\right] f(r)+V_{C}(r)
$$

with $f(r)=[1+\exp (r-R) / a]^{-1}$ and the Coulomb potential $V_{C}(r)$ for a proton. The interaction between nucleons, $V_{N N}$, is given by

$$
\begin{array}{r}
V_{n n}\left(\boldsymbol{r}, \boldsymbol{r}^{\prime}\right)=v_{s} P_{s}\left[1-x_{s} f(r)\right] \delta\left(\boldsymbol{r}-\boldsymbol{r}^{\prime}\right), \\
V_{p n}\left(\boldsymbol{r}, \boldsymbol{r}^{\prime}\right)= \\
v_{s} P_{s}\left[1-x_{s} f(r)\right] \delta\left(\boldsymbol{r}-\boldsymbol{r}^{\prime}\right) \\
+v_{t} P_{t}\left[1-x_{t} f(r)\right] \delta\left(\boldsymbol{r}-\boldsymbol{r}^{\prime}\right)
\end{array}
$$

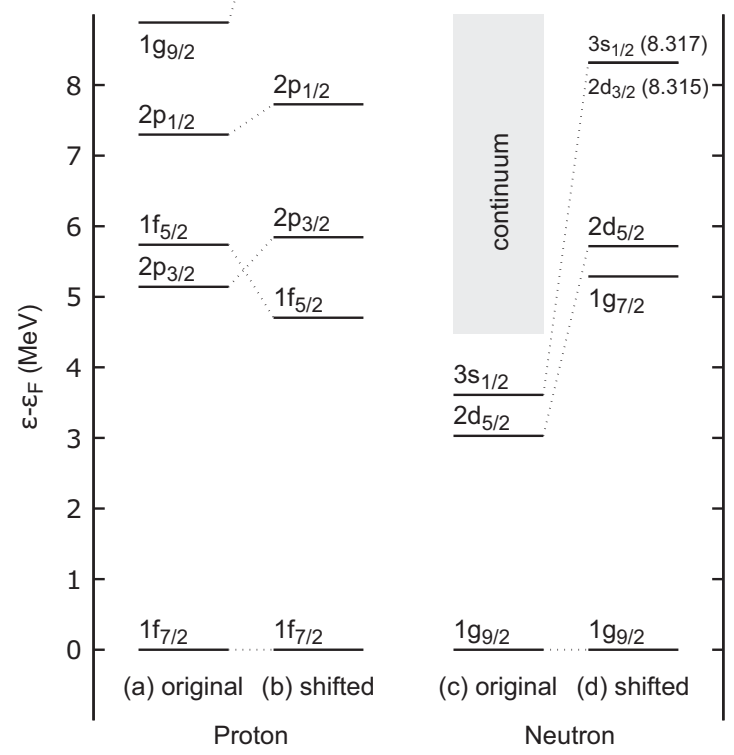

FIG. 1. Single-particle energies of the valence nucleons relative to the Fermi levels of the ${ }^{78} \mathrm{Ni}$ core. Shown are the proton single-particle states with the (a) original and (b) shifted $(-40 \mathrm{MeV})$ Woods-Saxon potentials, respectively, while the neutron single-particle states are depicted in (c) and (d). The shaded area represents the continuum region of neutron states. Notice that the $2 d_{3 / 2}$ and $3 s_{1 / 2}$ states in case (d) are almost degenerate, which are located at 8.315 and $8.317 \mathrm{MeV}$, respectively.

for neutron-neutron and proton-neutron, respectively, where $P_{S}$ and $P_{t}$ is a projector onto the $S=0$ and $S=1$ two-nucleon state, respectively.

\section{B. Numerical procedures}

The parameters of the Woods-Saxon potential are the standard one given in p. 239 of Ref. [43]. The Coulomb potential for a proton is obtained for the uniform charge distribution with the radius $R$. The single-particle states in the WoodsSaxon potential are obtained in a spherical box of $30 \mathrm{fm}$. The continuum states are then discretized. When diagonalizing the Hamiltonian (1), the single-particle states are truncated with a cut-off energy at $10 \mathrm{MeV}$, which is enough to cover a few major shells above the Fermi level that are relevant to lowlying states. The strength of the $S=0$ pair interaction $v_{s}$ is set as $-500 \mathrm{MeV} \mathrm{fm}^{3}$ for the qualitative discussion. We note that, with the present setup, the ground-state binding energy of the three-body system ${ }^{78} \mathrm{Ni}+2 n$ is $4.0 \mathrm{MeV}$, which is comparable to the estimated value of $4.5 \mathrm{MeV}[44,45]$. To see the effect of the $S=1$ pair interaction, $v_{t}$ is varied by multiplying a factor $f_{N N}$ as $v_{t}=f_{N N} \times v_{s}$. The parameters $x_{s}$ and $x_{t}$ are set as 0.5 , corresponding to the so-called mixed-type pair interaction.

Shown in Fig. 1 are the single-particle states near the Fermi levels relevant to the discussion below. 


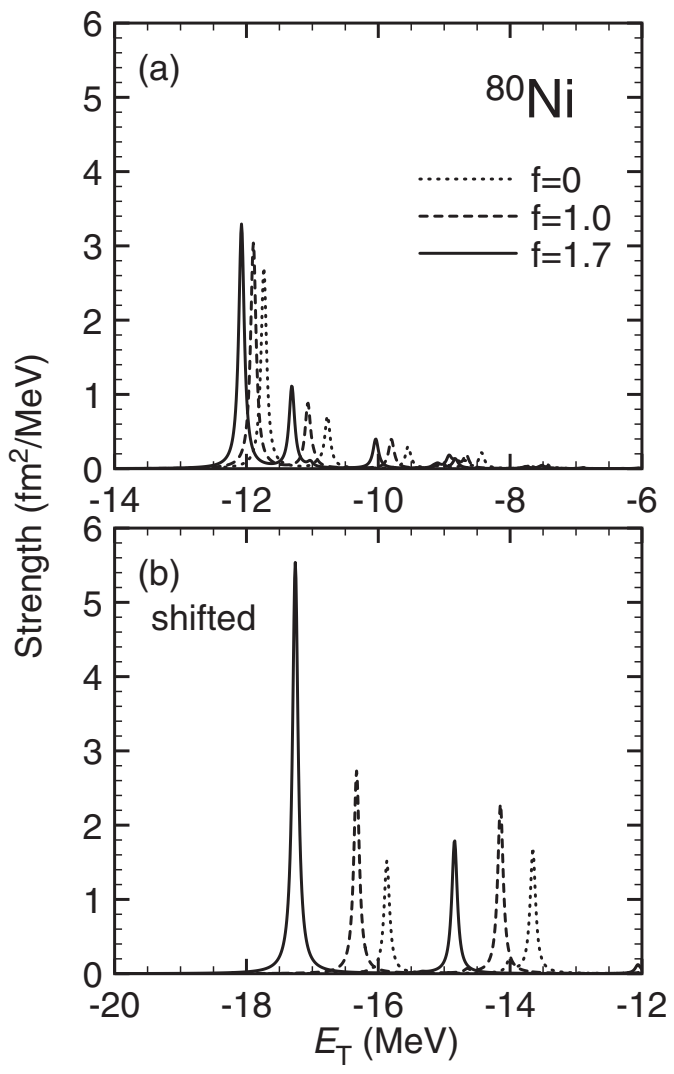

FIG. 2. (a) Calculated distributions for the SD $1^{-}$transition as functions of the excitation energy with respect to the target nucleus. The smearing parameter $\gamma=0.1 \mathrm{MeV}$ is used. The result obtained with $f_{N N}=0,1.0$, and 1.7 is depicted by the dotted, dashed, and solid lines, respectively. (b) Results obtained by changing the depth of the WS potential by $-40 \mathrm{MeV}$.

\section{Low-lying SD states in ${ }^{80} \mathrm{Cu}$}

We consider the response to the SD operator in the $(p, n)$ channel defined by

$$
\begin{aligned}
F_{K}^{J}= & \frac{1}{\sqrt{2}} \sum_{s s^{\prime} t t^{\prime}} \int d \boldsymbol{r} r \psi^{\dagger}\left(\boldsymbol{r} s^{\prime} t^{\prime}\right) \psi(\boldsymbol{r} s t) \\
& \times\left\langle s^{\prime}\left|\left[Y_{1} \otimes \sigma\right]_{K}^{J}\right| s\right\rangle\left\langle t^{\prime}\left|\tau_{-1}\right| t\right\rangle,
\end{aligned}
$$

where $\sigma$ and $\tau=\left(\tau_{+1}, \tau_{0}, \tau_{-1}\right)$ denote the spherical components of the Pauli matrix of spin and isospin, and $\psi^{\dagger}(\boldsymbol{r} s t), \psi(\boldsymbol{r} s t)$ represent the nucleon field operators. The reduced transition probability is given as

$$
B\left(S D, J ; J_{i} \rightarrow J_{f}\right)=\frac{1}{2 J_{i}+1}\left|\left\langle f|| F^{J} \| i\right\rangle\right|^{2} .
$$

We show in Fig. 2(a) the calculated SD $J=1$ transition strengths in ${ }^{80} \mathrm{Ni} \rightarrow{ }^{80} \mathrm{Cu}$. We found that the $1^{-}$state is the lowest among the SD states. One can see a prominent peak around $E_{\mathrm{T}}=-12 \mathrm{MeV}$ and several states with a small transition strength. The prominent state corresponds to the low-lying $1^{-}$state appearing at $-11.5 \mathrm{MeV}$ in the selfconsistent calculation based on the nuclear EDF method [42]; see Fig. 5(b) there. It is also noted that the present model predicts a prominent GT state around $E_{\mathrm{T}}=-3 \mathrm{MeV}$, which is constructed mainly by the $v 2 d_{5 / 2} \rightarrow \pi 2 d_{5 / 2}$ excitation. This is compatible with the microscopic calculations [39,42], where the GT state appears at $\approx-4.5 \mathrm{MeV}$. This correspondence shows that the present three-body model describes well the low-lying states in a nucleus with a spherical core plus two valence nucleons. The $1^{-}$state around $-12 \mathrm{MeV}$ plays a crucial role in the isotopic dependence of the $\beta$-decay halflives in the $\mathrm{Ni}$ isotopes $[39,42]$. We are thus going to look into the microscopic structure of this $1^{-}$state.

The effect of the $S=1$ pair interaction is investigated. To this end, we vary the strength of the interaction. The result obtained with $f_{N N}=0,1.0$, and 1.7 is depicted by the dotted, dashed, and solid lines, respectively, in the figure. With these parameters, the calculated mass difference is $11.7,11.9$, and $12.1 \mathrm{MeV}$, respectively. These are comparable to the evaluated value $[44,45], 13.4 \mathrm{MeV}$. The role of the $S=1$ pair interaction depends on the state; the lowest-lying state is weakly affected by the interaction compared with the second peak. Looking into the details of these states, we try to understand the role of the $S=1$ pair interaction in the SD excitations.

The lowest and second peaks are constructed mainly by the $\nu 2 d_{5 / 2} \otimes \pi 2 p_{3 / 2}$ and $\nu 2 d_{5 / 2} \otimes \pi 1 f_{5 / 2}$ configurations, respectively. The shift in the energy and the enhancement in the transition strength are governed by the two-body matrix element

$$
\begin{gathered}
\left\langle a^{\prime} b^{\prime}, J\left|V_{S=1}\right| a b, J\right\rangle=R_{a^{\prime} b^{\prime} a b} \times A_{a^{\prime} b^{\prime} a b}^{J}, \\
R=v_{t} \int d r \frac{1}{r^{2}}\left[1-x_{t} f(r)\right] u_{a^{\prime}}^{*}(r) u_{b^{\prime}}^{*}(r) u_{a}(r) u_{b}(r), \\
A^{J}=\frac{1}{8 \pi} \hat{j}_{a^{\prime}} \hat{j}_{b^{\prime}} \hat{j}_{a} \hat{j}_{b}\left[\left(\begin{array}{ccc}
j_{a^{\prime}} & j_{b^{\prime}} & J \\
\frac{1}{2} & \frac{1}{2} & -1
\end{array}\right)\left(\begin{array}{ccc}
j_{a} & j_{b} & J \\
\frac{1}{2} & \frac{1}{2} & -1
\end{array}\right)\right. \\
+\delta_{\ell_{a^{\prime}}+\ell_{b^{\prime}}+J, \text { odd }} \delta_{\ell_{a}+\ell_{b}+J, \text { odd }}(-1)^{\ell_{a^{\prime}}+\ell_{a}+j_{b^{\prime}}-j_{b}} \\
\left.\times\left(\begin{array}{ccc}
j_{a^{\prime}} & j_{b^{\prime}} & J \\
\frac{1}{2} & -\frac{1}{2} & 0
\end{array}\right)\left(\begin{array}{ccc}
j_{a} & j_{b} & J \\
\frac{1}{2} & -\frac{1}{2} & 0
\end{array}\right)\right],
\end{gathered}
$$

where $\hat{j}=\sqrt{2 j+1}$, and $u_{a}(r)$ is the radial wave function of the single-particle orbital. The diagonal matrix element $\left(R \times A^{J}\right)$ of the $v 2 d_{5 / 2} \otimes \pi 2 p_{3 / 2}$ and $v 2 d_{5 / 2} \otimes \pi 1 f_{5 / 2}$ configurations is $-5.60 \times 0.05=-0.27 \mathrm{MeV}$ and $-3.60 \times 0.12=$ $-0.44 \mathrm{MeV}$, respectively in the case of $f_{N N}=1.7$. The difference in the diagonal matrix element accounts for a stronger effect of the $S=1$ pair interaction seen on the second peak. The angular part $A^{J}$ of the diagonal matrix element for $J^{\pi}=$ $1^{-}$reads

$$
\begin{aligned}
& A^{1^{-}}\left(\ell_{j_{>}},(\ell-1)_{j_{>}} ; \ell_{j_{>}},(\ell-1)_{j_{>}}\right)=\frac{1}{8 \pi} \frac{\ell(\ell+1)}{2 \ell+1}, \quad(10 \mathrm{a}) \\
& A^{1^{-}}\left(\ell_{j_{<}},(\ell-1)_{j_{>}} ; \ell_{j_{<}},(\ell-1)_{j_{>}}\right)=\frac{1}{2 \pi} \frac{\ell^{3}}{(2 \ell+1)(2 \ell-1)},
\end{aligned}
$$

$A^{1^{-}}\left(\ell_{j_{<}},(\ell-1)_{j_{<}} ; \ell_{j_{<}},(\ell-1)_{j_{<}}\right)=\frac{1}{8 \pi} \frac{\ell(\ell-1)}{2 \ell-1}$,

where $\ell_{j \gtrless}$ denotes the orbital angular momentum satisfying $j=\ell \pm \frac{1}{2}$. The proton-neutron configuration involving the $j_{>}$ and $j_{<}$orbitals, Eq. (10b), have the largest matrix element 
as far as $\ell$ of the configurations is the same. Thus, we see a more substantial effect of the $S=1$ pair interaction for the $v 2 d_{5 / 2} \otimes \pi 1 f_{5 / 2}$ configuration though the spatial part $R$ is slightly smaller due to the difference in the number of nodes. Notice that the spatial part is larger in the GT excitation because the GT operator does not change the spatial structure, and the angular part is as large as the SD excitations as given in the Appendix, leading to a stronger effect of the $S=1$ pair interaction in the GT excitation.

Another feature seen in Eq. (10) is that the configuration involving a high- $\ell$ orbital acquires a large matrix element. Thus, we can expect that the $S=1$ pair interaction significantly affects the configuration composed of the $v 1 g_{7 / 2}$ orbital. We are going to investigate this in the following subsection.

\section{Role of a high- $\ell$ orbital}

With the present parameters, the $v 1 g_{7 / 2}$ orbital is located far above the Fermi level and embedded in the continuum states. To see the role of this high- $\ell$ orbital in the SD excitations, we deepen the potential so that the $\nu 1 g_{7 / 2}$ orbital appears near the Fermi level. The single-particle levels with the shifted potential is also shown in Fig. 1.

Figure 2(b) shows the SD transition strengths. A prominent state in low energies is constructed mainly by the $v 1 g_{7 / 2} \otimes$ $\pi 1 f_{5 / 2}$ configuration. As expected, we see a strong effect of the $S=1$ pair interaction. Since the $\nu 1 g_{7 / 2}$ and $\pi 1 f_{5 / 2}$ orbitals have the same number of nodes, the spatial part is also large. The second peak in Fig. 2(b) is also strongly affected by the $S=1$ pair interaction. This state is generated predominantly by the $v 2 d_{5 / 2} \otimes \pi 1 f_{5 / 2}$ configuration, corresponding to the second peak in Fig. 2(a).

\section{DISCUSSION USING NUCLEAR DFT}

We have found that the collective shift in the low-lying SD states due to the residual interactions is mostly governed by the diagonal matrix element. The larger the spatial and angular parts of the matrix element, the more strongly the $S=1$ pair interaction affects the SD states. For a larger spatial overlap, the orbitals should have the same number of nodes. High- $\ell$ orbitals acquire a large matrix element in the angular part.

Since the SD states are generated by not only the pair interactions but the spin-isospin interactions discarded in the model study above, we employ the nuclear density-functional theory (DFT) as a realistic calculation and try to fortify the finding obtained by the three-body model. Here we extend our discussion to the $\mathrm{SD} 0^{-}$and $2^{-}$states.

\section{A. Nuclear EDF method}

We perform a self-consistent Kohn-Sham-Bogoliubov (KSB) and the proton-neutron quasiparticle-random-phase approximation ( $p n \mathrm{QRPA}$ ) calculation. The details of the calculation scheme are found in Refs. [46] and [25,47] for the $\mathrm{KSB}$ and $p n \mathrm{QRPA}$, respectively. In brief, we solve the KSB equation in the coordinate space using cylindrical coordinates $\boldsymbol{r}=(\rho, z, \phi)$ with a mesh size of $\Delta \rho=\Delta z=0.6 \mathrm{fm}$ and a box boundary condition at $\left(\rho_{\max }, z_{\max }\right)=(14.7,14.4) \mathrm{fm}$. The quasiparticle (qp) states are truncated according to the qp energy cutoff at $60 \mathrm{MeV}$, and the qp states up to the magnetic quantum number $\Omega=23 / 2$ with positive and negative parities are included, with $\Omega$ being the $z$ component of the angular momentum. We introduce the truncation for the two-quasiparticle (2qp) configurations in the QRPA calculations, in terms of the 2qp energy as $60 \mathrm{MeV}$. For the normal (particle-hole) part of the EDF, we employ the SGII functional [48]. For the pairing (particle-particle, p-p) energy, we adopt the one in Ref. [49] that depends on both the IS and IV densities, in addition to the pair density, with the parameters given in Table III of Ref. [49]. The same pairing EDF is employed for the $S=0$ $p n$ pairing in the $p n \mathrm{QRPA}$ calculation, while the linear term in the IV density is dropped. The strength of the $S=1 p n$-pair interaction is varied by multiplying by a factor $f_{N N}$. It is noted that the calculation with a factor $f_{N N}=1.0$ describes well the low-lying GT states in ${ }^{18} \mathrm{~F}$ and ${ }^{42} \mathrm{Sc}$ [32-34]. Therefore, in the present investigation, we discuss the effect of the $S=1$ pairing by setting $f_{N N}=0$ (without $S=1$ pairing) and $f_{N N}=1.0$ (with $S=1$ pairing). The SD transition matrix elements are calculated as in Ref. [50].

\section{B. SD $0^{-}$state in ${ }^{132,134} \mathrm{Nb}$}

Among the SD excitations, the angular part of the diagonal matrix element is the largest in the $J=0$ transition; see Eq. (A2) in the Appendix. Therefore, we can expect a strong effect of the $S=1$ pair interaction to appear in the SD $0^{-}$ state involving such as $v 1 g_{7 / 2} \otimes \pi 1 f_{7 / 2}$ or $v 1 h_{9 / 2} \otimes \pi 1 g_{9 / 2}$ configuration. They correspond to the neutron-rich regions around $\mathrm{Sc}$ and $\mathrm{Nb}$ isotopes near the drip line. Since the neuron $1 g_{7 / 2}$ orbital is located above the $2 d_{5 / 2}$ orbital with the SGII functional, ${ }^{78} \mathrm{Ca}$ is a candidate for the study. However, we find it unbound. Therefore, we are going to investigate the neutron-rich $\mathrm{Zr}$ isotopes as an example.

The ground state of ${ }^{132,134} \mathrm{Zr}$ is calculated to be spherical, though we have local minima at finite deformation. The neutron occupation probability of the $1 h_{9 / 2}$ orbital is 0.04 and 0.06 in ${ }^{132} \mathrm{Zr}$ and ${ }^{134} \mathrm{Zr}$, respectively. Figure 3 shows the SD $0^{-}$transition-strength distribution in the thus calculated ${ }^{132,134} \mathrm{Zr}$. The low-energy peak is predominantly constructed by the $v 1 h_{9 / 2} \otimes \pi 1 g_{9 / 2}$ configuration. Due to the repulsive character of the spin-isospin residual interactions, the $2 \mathrm{qp}$ excitation of $v 1 h_{9 / 2} \otimes \pi 1 g_{9 / 2}$ is shifted higher in energy when $f_{N N}=0$. However, a tiny difference between the QRPA energy and the unperturbed one indicates the weak collectivity of the low-lying state at $E_{\mathrm{T}}=-15.2(-16.0) \mathrm{MeV}$ in ${ }^{132} \mathrm{Zr}$ $\left({ }^{134} \mathrm{Zr}\right)$. The transition strengths in a low-energy region are reduced due to the spin-isospin residual interactions, and they are brought into the giant resonance.

Since the pairing matrix elements entering into the QRPA equation are approximately proportional to $и и и и$ or $v v v v$ of the Bardeen-Cooper-Schrieffer amplitude [51], the p-p type or hole-hole type excitation acquires a large matrix element. Therefore, the low-lying SD $0^{-}$state here is strongly affected by the dynamical pair interaction. Indeed, the $S=1$ pair interaction lowers the energy and enhances the strength of the $\mathrm{SD} 0^{-}$state, as predicted based on the finding above. We can expect the $\beta$-decay rate in the $\mathrm{Zr}$ isotopes near the drip line is sensitively determined by the $S=1$ pair interaction through 


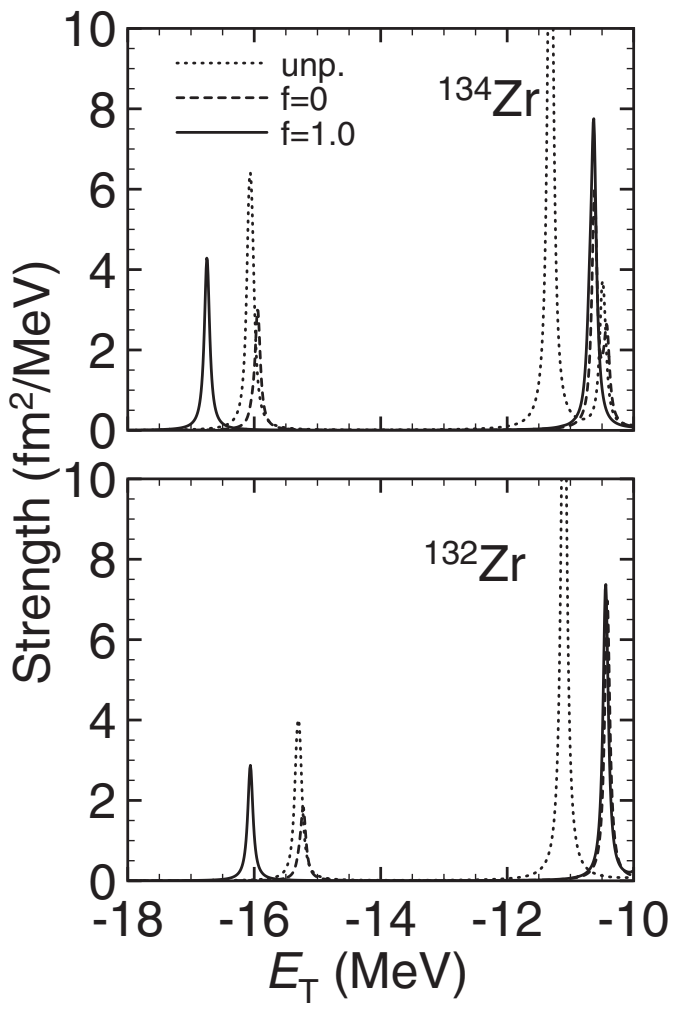

FIG. 3. Similar to Fig. 2 but obtained by employing the Skyrme$\mathrm{KSB}+p n \mathrm{QRPA}$ with the SGII functional for the SD $0^{-}$transition in ${ }^{132,134} \mathrm{Zr} \rightarrow{ }^{132,134} \mathrm{Nb}$. The results obtained with $f_{N N}=0$ and 1.0 are depicted by the dashed and solid lines, respectively. Shown is also the unperturbed strengths by the dotted line.

the first-forbidden transition because the positive-parity states, involving the $\pi 1 h_{11 / 2}$ orbital, show up in relatively higher excitation energy. It should be noted that extracting the details of nuclear-structure information from the $\beta$-decay rate with such a high $Q_{\beta}$ value requires a careful treatment of the Coulomb potential [52].

\section{SD 2- state in ${ }^{120,122} \mathrm{Sb}$ and ${ }^{68,70} \mathrm{Cu}$}

The SD $2^{-}$states appear in low energy widely in the nuclear chart [53]. It has thus been investigated as a unique first-forbidden $\beta$ decay [21], and is proposed as a probe of the physics beyond the standard model [54]. A neutron intruder orbital plays a decisive role in the occurrence of the low-lying $2^{-}$state. From Eq. (A3c), we can expect a strong effect of the $S=1$ pair interaction for the configurations such as $\nu 1 f_{7 / 2} \otimes \pi 1 d_{3 / 2}, \nu 1 g_{9 / 2} \otimes \pi 1 f_{5 / 2}, \nu 1 h_{11 / 2} \otimes \pi 1 g_{7 / 2}$, and $v 1 i_{13 / 2} \otimes \pi 1 h_{9 / 2}$.

Around ${ }^{120} \mathrm{Sn}$, the $\nu 1 h_{11 / 2}$ and $\pi 1 g_{7 / 2}$ orbitals are located near the Fermi level. The neutron occupation probability of the $1 h_{11 / 2}$ orbital is calculated to be $0.28(0.37)$ in ${ }^{120} \mathrm{Sn}$ $\left({ }^{122} \mathrm{Sn}\right)$. Similarly, the $\nu 1 g_{9 / 2}$ and $\pi 1 f_{5 / 2}$ orbitals are placed near the Fermi level around ${ }^{68} \mathrm{Ni}$. The neutron occupation probability of the $1 g_{9 / 2}$ orbital is $0.09(0.23)$ in ${ }^{68} \mathrm{Ni}\left({ }^{70} \mathrm{Ni}\right)$ in the present calculation.

Figure 4 shows the calculated transition-strength distribution in ${ }^{120,122} \mathrm{Sn}$ and ${ }^{68,70} \mathrm{Ni}$. The low-lying SD $2^{-}$state is
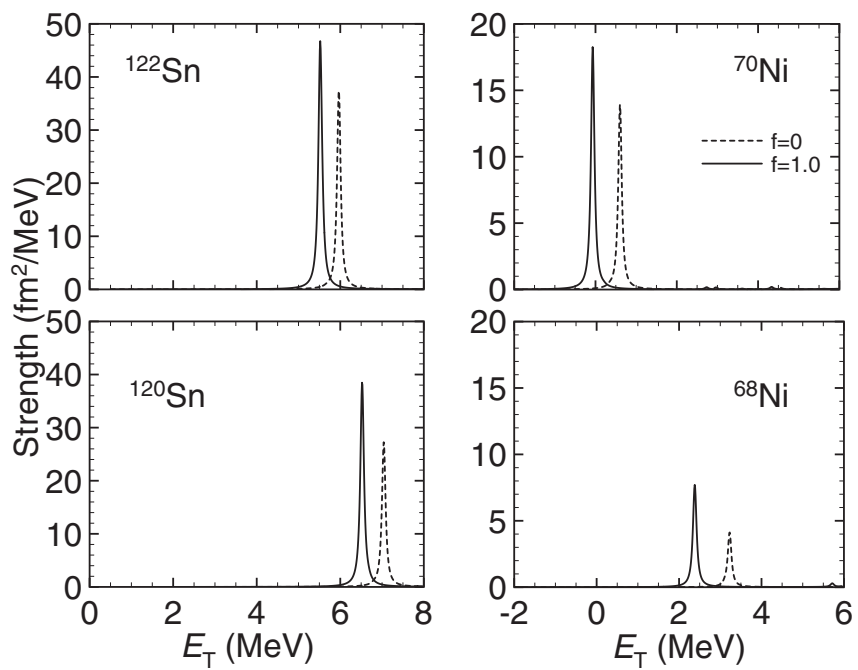

FIG. 4. Same as Fig. 3 but for the SD $2^{-}$transition in ${ }^{120,122} \mathrm{Sn} \rightarrow$ ${ }^{120,122} \mathrm{Sb}$ and ${ }^{68,70} \mathrm{Ni} \rightarrow{ }^{68,70} \mathrm{Cu}$.

dominantly generated by a p-p type excitation of $v 1 h_{11 / 2} \otimes$ $\pi 1 g_{7 / 2}$ and $\nu 1 g_{9 / 2} \otimes \pi 1 f_{5 / 2}$ in ${ }^{120,122} \mathrm{Sn}$ and ${ }^{68,70} \mathrm{Ni}$, respectively. We can see the strongest effect in ${ }^{68} \mathrm{Ni}$ among these examples. This is because the 2qp excitation constructing the SD $2^{-}$state is the most p-p type excitation; the amplitude ииии is the largest, giving a large diagonal matrix element.

\section{SUMMARY}

We have investigated the effect of the pn-pair interaction in the $T=0, S=1$ channel on the low-lying spin-dipole (SD) transitions. We aimed at clarifying the distinction of the role in between the SD and GT transitions. To this end, we have performed a three-body model calculation for the transition ${ }^{80} \mathrm{Ni} \rightarrow{ }^{80} \mathrm{Cu}$, where ${ }^{80} \mathrm{Ni}={ }^{78} \mathrm{Ni}+n+n$ and ${ }^{80} \mathrm{Cu}=$ ${ }^{78} \mathrm{Ni}+p+n$. The strength of the $S=1 p n$-pair interaction was varied to see the effect on the SD transition-strength distributions. The depth of the mean-field potential was also changed to study the shell effect. The effect of the $S=1$ $p n$-pair interaction depends on the spatial overlap of the $p n$ pair and the angular momentum of the valence nucleons. The $S=1 p n$-pair interaction in the SD excitations is active even if the spatial overlap of the $p n$ pair is weak, where the principal quantum numbers of the single-particle orbital are different by up to one unit, while only the $\Delta N=0$ excitation is allowed for the GT transition. The effect of the $S=1 p n$-pair interaction on the SD transition is thus weaker than on the GT transition.

To fortify the finding obtained by the three-body model analysis, we have performed the nuclear DFT calculations. In nuclei where the high $\ell$ orbitals are located close to the Fermi level, we have found a strong effect of the $S=1 p n$-pair interaction. In neutron-rich nuclei, the negative-parity states appear in low energies and thus the $\beta$-decay rate can be sensitive to the $S=1 p n$-pair interaction, similarly for the GT transition. A careful analysis of the forbidden $\beta$ decay is thus needed. 


\section{ACKNOWLEDGMENTS}

Discussions with K. Hagino and H. Sagawa are acknowledged. This work was supported by the JSPS KAKENHI (Grants No. JP19K03824, No. JP19K03861, and No. JP19K03872) and the JSPS/NRF/NSFC A3 Foresight Program "Nuclear Physics in the 21st Century." The nuclear DFT calculations were performed on Yukawa-21 at the Yukawa Institute for Theoretical Physics, Kyoto University.

\section{APPENDIX: ANGULAR PART OF DIAGONAL MATRIX ELEMENT}

We summarize here the angular part (9) of the diagonal matrix element of the $S=1$ pair interaction (4). For the GT operator, we have

$$
\begin{aligned}
& A^{1^{+}}\left(\ell_{j_{>}}, \ell_{j_{>}} ; \ell_{j_{>}}, \ell_{j_{>}}\right)=\frac{1}{4 \pi} \frac{(\ell+1)\left[2(\ell+1)^{2}+1\right]}{(2 \ell+3)(2 \ell+1)}, \\
& A^{1^{+}}\left(\ell_{j_{>}}, \ell_{j_{<}} ; \ell_{j_{>}}, \ell_{j_{<}}\right)=\frac{1}{8 \pi} \frac{3 \ell(\ell+1)}{2 \ell+1}, \\
& A^{1^{+}}\left(\ell_{j_{<}}, \ell_{j_{<}} ; \ell_{j_{<}}, \ell_{j_{<}}\right)=\frac{1}{4 \pi} \frac{\ell\left(2 \ell^{2}+1\right)}{(2 \ell+1)(2 \ell-1)} .
\end{aligned}
$$

For the SD operators, we have

$$
A^{0^{-}}\left(\ell_{j_{<}},(\ell-1)_{j_{>}} ; \ell_{j_{<}},(\ell-1)_{j_{>}}\right)=\frac{\ell}{4 \pi}
$$

and

$$
\begin{aligned}
& A^{2^{-}}\left(\ell_{j_{\succ}},(\ell-1)_{j_{\supset}} ; \ell_{j_{\supset}},(\ell-1)_{j_{\supset}}\right) \\
& =\frac{1}{8 \pi} \frac{\left[(2 \ell+1)^{2}+6\right](\ell+1) \ell}{(2 \ell+3)(2 \ell+1)(2 \ell-1)}, \\
& A^{2^{-}}\left(\ell_{j_{<}},(\ell-1)_{j_{\supset}} ; \ell_{j_{<}},(\ell-1)_{j_{\supset}}\right) \\
& =\frac{1}{4 \pi} \frac{\ell(\ell+1)(\ell-1)}{(2 \ell+1)(2 \ell-1)}, \\
& A^{2^{-}}\left(\ell_{j_{\succ}},(\ell-1)_{j_{<}} ; \ell_{j_{\supset}},(\ell-1)_{j_{<}}\right) \\
& =\frac{1}{8 \pi} \frac{5 \ell(\ell+1)(\ell-1)}{(2 \ell+1)(2 \ell-1)}, \\
& A^{2^{-}}\left(\ell_{j_{<}},(\ell-1)_{j_{<}} ; \ell_{j_{<}},(\ell-1)_{j_{<}}\right) \\
& =\frac{1}{8 \pi} \frac{\left[(2 \ell-1)^{2}+6\right] \ell(\ell-1)}{(2 \ell+1)(2 \ell-1)(2 \ell-3)}
\end{aligned}
$$

for $J=0$ and $J=2$, respectively.
[1] D. M. Brink and R. A. Broglia, Nuclear Superfluidity: Pairing in Finite Systems, Cambridge Monographs on Particle Physics, Nuclear Physics and Cosmology (Cambridge University Press, Cambridge, 2005).

[2] J. Schrieffer, Theory of Superconductivity, Frontiers in Physics (W.A. Benjamin, Reading, MA, 1964).

[3] A. B. Migdal, Two interacting particles in a potential well, Yad. Fiz. 16, 427 (1972) [Sov. J. Nucl. Phys. 16, 238 (1973)].

[4] G. F. Bertsch and H. Esbensen, Pair correlations near the neutron drip line, Ann. Phys. (NY) 209, 327 (1991).

[5] M. Zhukov, B. Danilin, D. Fedorov, J. Bang, I. Thompson, and J. Vaagen, Bound state properties of Borromean halo nuclei: ${ }^{6} \mathrm{He}$ and ${ }^{11} \mathrm{Li}$, Phys. Rep. 231, 151 (1993).

[6] F. V. De Blasio, M. Hjorth-Jensen, O. Elgaroy, L. Engvik, G. Lazzari, M. Baldo, and H. J. Schulze, Coherence length of neutron superfluids, Phys. Rev. C 56, 2332 (1997).

[7] M. Matsuo, K. Mizuyama, and Y. Serizawa, Di-neutron correlation and soft dipole excitation in medium mass neutron-rich nuclei near drip-line, Phys. Rev. C 71, 064326 (2005).

[8] M. Matsuo, Spatial structure of neutron Cooper pair in low density uniform matters, Phys. Rev. C 73, 044309 (2006).

[9] K. Hagino and H. Sagawa, Pairing correlations in nuclei on the neutron-drip line, Phys. Rev. C 72, 044321 (2005).

[10] N. Pillet, N. Sandulescu, and P. Schuck, Generic strong coupling behavior of Cooper pairs in the surface of superfluid nuclei, Phys. Rev. C 76, 024310 (2007).

[11] T. Nakamura, A. M. Vinodkumar, T. Sugimoto, N. Aoi, H. Baba, D. Bazin, N. Fukuda, T. Gomi, H. Hasegawa, N. Imai, M. Ishihara, T. Kobayashi, Y. Kondo, T. Kubo, M. Miura, T. Motobayashi, H. Otsu, A. Saito, H. Sakurai, S. Shimoura et al., Observation of Strong Low-lying E1 Strength in the Two-Neutron Halo Nucleus ${ }^{11}$ Li, Phys. Rev. Lett. 96, 252502 (2006).
[12] Y. Kubota, A. Corsi, G. Authelet, H. Baba, C. Caesar, D. Calvet, A. Delbart, M. Dozono, J. Feng, F. Flavigny, J.-M. Gheller, J. Gibelin, A. Giganon, A. Gillibert, K. Hasegawa, T. Isobe, Y. Kanaya, S. Kawakami, D. Kim, Y. Kikuchi et al., Surface Localization of the Dineutron in ${ }^{11} \mathrm{Li}$, Phys. Rev. Lett. 125, 252501 (2020).

[13] S. Frauendorf and A. O. Macchiavelli, Overview of neutronproton pairing, Prog. Part. Nucl. Phys. 78, 24 (2014).

[14] K. Yoshida, Proton-neutron pairing vibrations in $N=Z$ nuclei: Precursory soft mode of isoscalar pairing condensation, Phys. Rev. C 90, 031303(R) (2014).

[15] E. Litvinova, C. Robin, and I. A. Egorova, Soft modes in the proton-neutron pairing channel as precursors of deuteron condensate in $N=Z$ nuclei, Phys. Lett. B 776, 72 (2018).

[16] Y. Chazono, K. Yoshida, K. Yoshida, and K. Ogata, Proton induced deuteron knockout reaction as a probe of an isoscalar proton-neutron pair in nuclei, Phys. Rev. C 103, 024609 (2021).

[17] K. Yoshida, Isovector spin susceptibility: Isotopic evolution of collectivity in spin response, Phys. Rev. C 104, 014309 (2021).

[18] J. Engel, P. Vogel, and M. R. Zirnbauer, Nuclear structure effects in double beta decay, Phys. Rev. C 37, 731 (1988).

[19] J. Suhonen, A. Faessler, T. Taigel, and T. Tomoda, Suppression of the $\beta^{+}$-decays of ${ }^{148} \mathrm{Dy},{ }^{150} \mathrm{Er}$ and ${ }^{152} \mathrm{Yb}$, Phys. Lett. B 202, 174 (1988).

[20] J. Suhonen, T. Taigel, and A. Faessler, pnQRPA calculation of the $\beta^{+} / \mathrm{EC}$ quenching for several neutron-deficient nuclei in mass regions $A=94-110$ and $A=146-156$, Nucl. Phys. A 486, 91 (1988).

[21] H. Homma, E. Bender, M. Hirsch, K. Muto, H. V. KlapdorKleingrothaus, and T. Oda, Systematic study of nuclear beta decay, Phys. Rev. C 54, 2972 (1996). 
[22] I. N. Borzov, S. A. Fayans, E. Krömer, and D. Zawischa, Ground state properties and $\beta$-decay half-lives near ${ }^{132} \mathrm{Sn}$ in a self-consistent theory, Z. Phys. A 355, 117 (1996).

[23] J. Engel, M. Bender, J. Dobaczewski, W. Nazarewicz, and R. Surman, Beta decay of $r$ process waiting point nuclei in a selfconsistent approach, Phys. Rev. C 60, 014302 (1999).

[24] I. N. Borzov and S. Goriely, Weak interaction rates of neutronrich nuclei and the $r$-process nucleosynthesis, Phys. Rev. C 62 , 035501 (2000).

[25] K. Yoshida, Spin-isospin response of deformed neutronrich nuclei in a self-consistent Skyrme energy-densityfunctional approach, Prog. Theor. Exp. Phys. 2013, 113D02 (2013).

[26] K. Yoshida, Charge-exchange modes of excitation in deformed neutron-rich nuclei, AIP Conf. Proc. 1681, 050006 (2015).

[27] K. Yoshida, Spin-isospin responses of deformed neutron-rich nuclei, PoS INPC2016, 059 (2017).

[28] Y. Tanimura, H. Sagawa, and K. Hagino, Three-body model calculations for $N=Z$ odd-odd nuclei with $T=0$ and $T=1$ pairing correlations, Prog. Theor. Exp. Phys. 2014, 053 D02 (2014).

[29] Y. Tanimura and H. Sagawa, Three-body model for an isoscalar spin-triplet neutron-proton pair in ${ }^{102} \mathrm{Sb}$, Phys. Rev. C 93, 064319 (2016).

[30] F. Minato and Y. Tanimura, Spin-isospin properties of $N=Z$ odd-odd nuclei from a core $+p n$ three-body model including core excitations, Eur. Phys. J. A 56, 45 (2020).

[31] C. L. Bai, H. Sagawa, G. Colò, Y. Fujita, H. Q. Zhang, X. Z. Zhang, and F. R. Xu, Low-energy collective Gamow-Teller states and isoscalar pairing interaction, Phys. Rev. C 90, 054335 (2014).

[32] H. Fujita, Y. Fujita, Y. Utsuno, K. Yoshida, T. Adachi, A. Algora, M. Csatlós, J. M. Deaven, E. Estevez-Aguado, C. J. Guess, J. Gulyás, K. Hatanaka, K. Hirota, R. Hutton, D. Ishikawa, A. Krasznahorkay, H. Matsubara, F. Molina, H. Okamura, H. J. Ong et al., Experimental study of GamowTeller transitions via the high-energy-resolution ${ }^{18} \mathrm{O}\left({ }^{3} \mathrm{He}, t\right){ }^{18} \mathrm{~F}$ reaction: Identification of the low-energy "super" -GamowTeller state, Phys. Rev. C 100, 034618 (2019).

[33] Y. Fujita, H. Fujita, T. Adachi, C. L. Bai, A. Algora, G. P. A. Berg, P. von Brentano, G. Colò, M. Csatlós, J. M. Deaven, E. Estevez-Aguado, C. Fransen, D. De Frenne, K. Fujita, E. Ganioğlu, C. J. Guess, J. Gulyás, K. Hatanaka, K. Hirota, M. Honma et al., Observation of Low- and High-Energy GamowTeller Phonon Excitations in Nuclei, Phys. Rev. Lett. 112, 112502 (2014).

[34] Y. Fujita, H. Fujita, T. Adachi, G. Susoy, A. Algora, C. L. Bai, G. Colò, M. Csatlós, J. M. Deaven, E. Estevez-Aguado, C. J. Guess, J. Gulyás, K. Hatanaka, K. Hirota, M. Honma, D. Ishikawa, A. Krasznahorkay, H. Matsubara, R. Meharchand, F. Molina et al., High-resolution study of Gamow-Teller excitations in the ${ }^{42}\left(\mathrm{Ca}^{3} \mathrm{He}, t\right)^{42} \mathrm{Sc}$ reaction and the observation of a "low-energy super-Gamow-Teller state", Phys. Rev. C 91, 064316 (2015).

[35] K. Yoshida, Charge-exchange dipole excitations in neutron-rich nuclei: $-1 \hbar \omega_{0}$, anti-analog pygmy and anti-analog giant resonances, Phys. Rev. C 96, 051302(R) (2017).
[36] I. N. Borzov, Gamow-Teller and first-forbidden decays near the $r$-process paths at $N=50,82$, and 126, Phys. Rev. C 67, 025802 (2003).

[37] M. T. Mustonen and J. Engel, Global description of $\beta^{-}$decay in even-even nuclei with the axially-deformed Skyrme finiteamplitude method, Phys. Rev. C 93, 014304 (2016).

[38] T. Marketin, L. Huther, and G. Martínez-Pinedo, Large-scale evaluation of $\beta$-decay rates of $r$-process nuclei with the inclusion of first-forbidden transitions, Phys. Rev. C 93, 025805 (2016).

[39] I. N. Borzov, Global calculations of beta-decay properties based on the Fayans functional, Phys. At. Nucl. 83, 700 (2020).

[40] M. Bender, P.-H. Heenen, and P.-G. Reinhard, Self-consistent mean-field models for nuclear structure, Rev. Mod. Phys. 75, 121 (2003).

[41] T. Nakatsukasa, K. Matsuyanagi, M. Matsuo, and K. Yabana, Time-dependent density-functional description of nuclear dynamics, Rev. Mod. Phys. 88, 045004 (2016).

[42] K. Yoshida, Suddenly shortened half-lives beyond ${ }^{78} \mathrm{Ni}: N=$ 50 magic number and high-energy non-unique first-forbidden transitions, Phys. Rev. C 100, 024316 (2019).

[43] A. Bohr and B. Mottelson, Nuclear Structure: Single-Particle Motion, Nuclear Structure (Benjamin, Reading, MA, 1969).

[44] W. Huang, M. Wang, F. Kondev, G. Audi, and S. Naimi, The AME 2020 atomic mass evaluation (I). Evaluation of input data, and adjustment procedures, Chin. Phys. C 45, 030002 (2021).

[45] M. Wang, W. Huang, F. Kondev, G. Audi, and S. Naimi, The AME 2020 atomic mass evaluation (II). Tables, graphs and references, Chin. Phys. C 45, 030003 (2021).

[46] H. Kasuya and K. Yoshida, Hartree-Fock-Bogoliubov theory for odd-mass nuclei with a time-odd constraint and application to deformed halo nuclei, Prog. Theor. Exp. Phys. 2021, 013D01 (2021)

[47] K. Yoshida, Erratum: Spin-isospin response of deformed neutron-rich nuclei in a self-consistent Skyrme energy-densityfunctional approach, Prog. Theor. Exp. Phys. 2021, 019201 (2021).

[48] N. van Giai and H. Sagawa, Spin-isospin and pairing properties of modified Skyrme interactions, Phys. Lett. B 106, 379 (1981).

[49] M. Yamagami, Y. R. Shimizu, and T. Nakatsukasa, Optimal pair density functional for description of nuclei with large neutron excess, Phys. Rev. C 80, 064301 (2009).

[50] K. Yoshida, Charge-exchange dipole excitations in deformed nuclei, Phys. Rev. C 102, 054336 (2020).

[51] P. Ring and P. Schuck, The Nuclear Many-Body Problem, Texts and Monographs in Physics (Springer-Verlag, Berlin, 1980).

[52] W. Horiuchi, T. Sato, Y. Uesaka, and K. Yoshida, Electron wave functions in beta-decay formulas revisited (I): Gamow-Teller and spin-dipole contributions to allowed and first-forbidden transitions, Prog. Theor. Exp. Phys., ptab069 (2021).

[53] F. Didierjean and G. Walter, Distribution of $\log f t$ values for allowed, first and second forbidden beta transitions $(10<A \leqslant 100) \quad$ (1994), https://cds.cern.ch/record/261858/ files/P00022752.pdf.

[54] A. Glick-Magid, Y. Mishnayot, I. Mukul, M. Hass, S. Vaintraub, G. Ron, and D. Gazit, Beta spectrum of unique first-forbidden decays as a novel test for fundamental symmetries, Phys. Lett. B 767, 285 (2017). 